Enfermagem Brasil 2018;17(2):90-8

\title{
ARTIGO ORIGINAL \\ Burnout y condiciones metabólicas en profesionales de enfermería: un estudio piloto
}

Magno Conceição das Merces, M.Sc. ${ }^{*}$ Antônio Marcos Tosoli Gomes, D.Sc. ${ }^{*}$, Else Lorena Pereira Guimarães ${ }^{* \star *}$, Amália Ivine Costa Santana, M.Sc. ${ }^{* * \star *}$, Douglas de Souza e Silva ${ }^{* * * * *}$, Yndira Yta Machado, M.Sc. ${ }^{\star \star * \star *}$, Pablo Luiz Santos Couto, M.Sc. ${ }^{* \star * * \star *}$, Luiz Carlos Moraes França, M.Sc.. ${ }^{* * * * * *}$, Virginia Paiva de Figueiredo, M.Sc. ${ }^{* * * * * * *}$, Argemiro D'Oliveira Júnior, D.Sc. ${ }^{* \star \star \star \star \star \star * \star *}$

*Biólogo e Enfermeiro, Professor do Departamento de Ciências da Vida, Universidade do Estado da Bahia (UNEB), Campus I, ${ }^{* \star E n f e r m e i r o, ~ P r o f e s s o r ~ T i t u l a r ~ d o ~ D e p a r t a m e n t o ~ d e ~}$ Enfermagem Médico-Cirúrgica e do Programa de Pós-graduação em Enfermagem da Faculdade de Enfermagem da Universidade do Estado do Rio de Janeiro (UERJ), ${ }^{* * *}$ Enfermeira graduada pela UNEB, Campus XII, ${ }^{* * * *}$ Enfermeira do Hospital Universitário Professor Edgard Santos da Universidade Federal da Bahia (UFBA), ${ }^{* * * * * E n f e r m e i r o, ~}$ Residente em Terapia Intensiva da UNEB, Campus I, ***** Enfermeira, Doutoranda em Enfermagem pelo Programa de Pós-graduação em Enfermagem da UERJ, ${ }^{* * * * * * \star}$ Enfermeiro, Mestre em Enfermagem pela Escola de Enfermagem da UFBA, ${ }^{* * * * * * *}$ Enfermeiro, Mestre em Enfermagem pelo Programa de Pós-Graduação em Enfermagem da UERJ, ${ }^{* * * * * * * \star}$ Enfermeira, Doutoranda em Enfermagem pelo Programa de Pós-graduação em Enfermagem da UERJ, *********Médico, Professor Titular de Clínica Médica e dos Programas de Pós-graduações em Ciências da Saúde e Medicina e Saúde da Faculdade de Medicina da Bahia da UFBA

Recebido em 15 de abril de 2018; aceito em 30 de abril de 2018.

Dirección para correspondencia: Magno Conceição das Merces, Rua Silveira Martins, 2555, Cabula 41150-000 Salvador, BA, E-mail: magnomerces@hotmail.com; Antônio Marcos Tosoli Gomes: mtosoli@gmail.com; Else Lorena Pereira Guimarães: Iorena_pg1@hotmail.com; Amália Ivine Costa Santana: amalia0807@gmail.com; Douglas de Souza e Silva: douglasssgbi@hotmail.com; Yndira Yta Machado: yndiramachado@gmail.com; Pablo Luiz Santos Couto: pabloluizsc@hotmail.com; Luiz Carlos Moraes França: Icmoraesfranca@hotmail.com; Virginia Paiva de Figueiredo: virginiafigueiredo@yahoo.com.br; Argemiro D'Oliveira Júnior: argemiro@ufba.br

\section{Resumen}

El objetivo fue estimar la asociación entre el Síndrome de Burnout (SB) y las condiciones metabólicas en profesionales de enfermería de la Atención Primaria de Salud (APS) de un municipio de Bahía, Brasil. Se trata de un estudio piloto de tipo transversal, conducido con profesionales de enfermería de la APS. Se aplicó un cuestionario que contenía secciones sociodemográficas, laborales, de estilo de vida y biología humana, en el periodo de enero a marzo del 2015. Para evaluar el SB se utilizó el Maslach Burnout Inventory - Human Services Survey, las condiciones metabólicas fueron medidas a través de cuestionamientos autorreferidos, medición de presión arterial, evaluaciones antropométricas y colecta de muestras sanguíneas. Los resultados evidenciaron una alta prevalencia de SB asociado con variables demográficas, metabólicas y biológicas. Al evidenciar el SB como un factor capaz de influenciar negativamente en la salud de los profesionales de enfermería, se busca contribuir con nuevas estrategias de intervención con énfasis en los factores de riesgos emergentes.

Palabras-clave: agotamiento profesional, profesionales de enfermería, síndrome x metabólica, atención primaria de salud.

\section{Abstract \\ Burnout and metabolic conditions are professionals of nursing: a pilot study}

The objective of this study was to compare between the Burnout Syndrome (SB) and some metabolic occupational therapy of the Primary Health Care (PHS) of Bahia, Brazil. It is a pilot, cross-sectional population pilot, conducted with PHS nursing professionals. A questionnaire was applied with sociodemographic, labor, lifestyle and human biology variables, from January to March, 2015. To evaluate SB, the Maslach Burnout Inventory was used as the conditions metabolic parameters were measured by self-referenced questionnaires, blood pressure 
measurements, anthropometric measurements and blood samples. They are evidenced by the high prevalence of SB and its associations with sociodemographic, metabolic and human biological variables. The evidence of SB as a factor capable of negatively influencing the health of nursing professionals seeks to contribute with new intervention strategies with an emphasis on emerging risk factors.

Key-words: professional exhaustion, nursing professionals, syndrome $\mathrm{x}$ metabolic, primary health care.

\section{Resumo}

Burnout e condições metabólicas em profissionais de enfermagem: um estudo piloto

O objetivo foi estimar a associação entre Síndrome de Burnout (SB) e condições metabólicas entre profissionais de enfermagem da Atenção Primária à Saúde (APS) de um município da Bahia, Brasil. Trata-se de estudo piloto, populacional de corte transversal, conduzido com profissionais de enfermagem da APS. Foi aplicado um questionário contento seções sociodemográficas, laborais, de estilo de vida e biologia humana, no período de janeiro a março de 2015. Para avaliar a SB utilizou-se o Maslach Burnout Inventory - Human Services Survey, as condições metabólicas foram aferidas mediante questionamentos autorreferidos, aferição de pressão arterial, avaliações antropométricas e coleta de amostras sanguíneas. Os resultados evidenciaram alta prevalência de $S B$ e sua associação com variáveis sociodemográficas, metabólicas e biologia humana. Ao evidenciar a SB como um fator capaz de influenciar negativamente a saúde dos profissionais de enfermagem, busca-se contribuir com novas estratégias de intervenção com ênfase nos fatores de risco emergentes.

Palavras-chave: esgotamento profissional, profissionais de enfermagem; síndrome metabólica, atenção primária à saúde.

Introducción

En los días hodiernos, el estrés ocupacional corresponde a un problema notorio de salud pública, considerando al número creciente de trastornos mentales y el comportamiento asociado a las actividades laborales que culminan finalmente en el alejamiento del trabajador de sus actividades, comprometiendo además la calidad de la asistencia y convirtiéndose en un hecho preocupante en los ámbitos organizacionales y sociales [1,2].

Las consecuencias fisiopatológicas del estrés ocupacional crónico pueden ocasionar el aparecimiento y curso de diversas enfermedades y síndromes, siendo una de estas el Síndrome de Burnout (SB). Este síndrome trae consigo repercusiones en el organismo del trabajador, sobretodo en el sistema cardiovascular, dado que posee una vasta participación en la adaptación a elevados niveles de estrés [3,4].

EI SB se caracteriza por presentar agotamiento emocional (AE), despersonalización (DP) y Realización Profesional Reducida (RPR) en el trabajo, "que ocurren frecuentemente en personas que ejecutan algún tipo de trabajo en el cual necesitan relacionarse con personas de forma próxima y directa". En este ámbito, los profesionales de salud son expuestos a estresores ocupacionales, representando el grupo de empleados más comprometidos, manifestando índices de burnout de 30 a 47\% [6].

En el área de salud en 1990, la enfermería fue clasificada por la Health Education Authority como la cuarta profesión más estresante en el campo público. La enfermería desarrolla sus prácticas laborales en diversos contextos. Aquí se destaca la atención primaria de salud (APS), la que tiene como objetivo ser la organización de puerta de entrada al sistema de salud, dando énfasis a las medidas de prevención, promoción y rehabilitación de la salud.

Los profesionales de enfermería que trabajan en la APS pasan por un proceso de frustración constante debido a algunos factores a seguir: el nivel de atención que debería tener como objetivo el fortalecimiento de las propuestas de prevención y promoción de la salud, el cual acaba teniendo más énfasis en condiciones curativas; precarización del trabajo; inconsistencia entre demanda y oferta de atendimientos; exceso de actividades gerenciales; ausencia de autonomía y otros.

Una investigación en Brasil entre profesionales de enfermería que trabajan en la atención primaria puntuó una asociación positiva entre SB y adiposidad abdominal. Otro estudio desarrollado en Israel muestra evidencias entre SB con problemas de salud, incluyendo el Síndrome Metabólico (SM), desregulación del eje hipotálamo-hipófisis- adrenal (HHA), juntamente con la activación del sistema nervioso simpático, disturbios del sueño, inflamación 
sistémica, funciones inmunocomprometidas, coagulación de la sangre, fibrinólisis y pésimos comportamientos de salud [7].

De acuerdo a la I Directriz Brasileña de Diagnóstico y Tratamiento del Síndrome Metabólico, el SM constituye "un trastorno complejo representado por un conjunto de factores de riesgo cardiovascular" [8:5], para diagnóstico del SM el paciente debe presentar una combinación de por lo menos tres de los cinco componentes: Circunferencia abdominal elevada, hipertrigliceridemia, reducción del HDL colesterol, hipertensión arterial sistémica y hiperglicemia [8].

Investigaciones en diversos países apuntan que el aumento del estrés en la vida cotidiana puede resultar en alteraciones fisiológicas y neuroendocrinas, una vez que las respuestas a los eventos estresantes están relacionadas como el eje HHA, que es responsable por las respuestas a la exposición al estrés crónico. Sin embargo, disfunciones en este eje y disturbios del ritmo circadiano predisponen a disturbios metabólicos como el SM, adiposidad abdominal y obesidad $[4,9,10]$.

Considerando los hechos expuestos y la escasez de estudios que apunten a la asociación entre SB y condiciones metabólicas en profesionales de enfermería, la presente investigación buscó por medio de un estudio piloto, estimar la asociación entre SB y condiciones metabólicas entre profesionales de enfermería de la APS de un municipio de Bahía, Brasil.

\section{Material y métodos}

Estudio piloto, poblacional de corte transversal, conducido en la APS de un municipio localizado en Bahía, Brasil. La colecta de datos sucedió en el periodo de enero a marzo del 2015. La población del estudio fue compuesta por 60 profesionales de enfermería que desarrollaban actividades asistenciales en la APS, siendo $38,3 \%$ de enfermeras y $61,7 \%$ de auxiliares y técnicas de enfermería.

Para el levantamiento de las variables fue aplicado un cuestionario elaborado por los autores del presente estudio, conteniendo secciones socio demográficas, laborales, de estilo de vida y biología humana. Para evaluar la SB en la población estudiada, se utilizó el Maslach Burnout Inventory - Human Services Survey (MBI - HSS) traducido y adaptado al portugués por Benevides-Pereira [11]. EI MBI - HSS posee 22 ítems que exploran aspectos del nivel de agotamiento emocional, despersonalización y reducida realización profesional. La forma de puntuación adoptada es representada en una escala de tipo Likert de cinco puntos, siendo "1" nunca, "2" - raramente, "3" - algunas veces, "4", - frecuentemente, e "5" - siempre. Después del sumatorio de las puntuaciones obtenidas por las dimensiones de la SB, se siguió la clasificación en niveles alto, medio y bajo, conforme los puntos de corte propuestos por Moreira et al. [12] (Tabla I).

Tabla I - Dimensiones del Síndrome de Burnout por el Maslach Burnout Inventory.

\begin{tabular}{lllll}
\hline \multirow{2}{*}{ Dimensiones } & \multicolumn{2}{l}{ Puntuaciones } & \multicolumn{2}{l}{ Preguntas } \\
\cline { 2 - 5 } & Alta & Media & Baja & \\
\hline EE & $\geq 27$ & $19-26$ & $<19$ & $1,2,3,6,8,13,14,16$ e 20 \\
\hline DP & $\geq 10$ & $6-9$ & $<6$ & $5,10,11,15$ e 22 \\
\hline RRP & $\leq 33$ & $34-39$ & $\geq 40$ & $4,7,9,12,17,18,19$ e 21 \\
\hline Fuen
\end{tabular}

En frente a la falta de consenso en la literatura para la interpretación del MBI, fueron utilizados los criterios de Grunfeld et al. [13] apuntando nivel alto en EE o DP, o nivel bajo en RRP de forma independiente, para ser considerado con SB.

Fue evaluada la confiabilidad interna de las categorías del instrumento MBI - HSS utilizando el coeficiente Alfa de Cronbach. Es sabido que los valores de alfa arriba de 0.70 indican consistencia interna aceptable, sosteniendo la confiabilidad del instrumento para este estudio. Se obtuvo 0.82 para EE, 0,79 para DP y 0,81 para RRP.

Para evaluar las condiciones metabólicas, fueron realizados cuestionamientos autorreferidos, medidas de presión arterial, evaluaciones antropométricas, tales como: Circunferencia abdominal, peso y altura. La medida de la presión arterial fue realizada en duplicado, en el miembro superior izquierdo, con el paciente sentado después de cinco minutos de reposo, utilizando la media de las dos últimas mediciones. Se calibró previamente el estetoscopio de la marca $($ Littmann®) y el esfigmomanómetro de la marca $(B D \AA)$. 
La circunferencia abdominal fue obtenida por una cinta inelástica, con capacidad de $150 \mathrm{~cm}$ y $1 \mathrm{~cm}$ para el grado de precisión de la marca ISP®, siendo la medida de la distancia entre el borde inferior del reborde costal y el ilíaco, en el plano horizontal, en posición ortoestática, brazos a lo largo del cuerpo, pies juntos, peso dividido entre las piernas y la mirada volcada para el horizonte. La medida del peso y la altura fueron autorreferidas.

Los profesionales fueron dirigidos a un único laboratorio, donde fueron colectadas las muestras sanguíneas, obtenidas en ayuno de 12 a 14 horas. Para las muestras séricas destinadas al estudio de la glicemia, HDL y triglicéridos, fueron utilizadas técnicas laboratoriales enzimáticas y colorimétricas convencionales.

Se consideraron condiciones metabólicas alteradas cuando el profesional presentaba: circunferencia abdominal $>88 \mathrm{~cm}$ y HDL-c $<50 \mathrm{mg} / \mathrm{dL}$ para mujeres y $<40 \mathrm{mg} / \mathrm{dL}$ para hombres, triglicéridos $\geq 150,0 \mathrm{mg} / \mathrm{dL}$, glicemia en ayuno $\geq 110,0 \mathrm{mg} / \mathrm{dL}$ y presión arterial $(\geq$ $130 / 85 \mathrm{mmHg}$ ). Para considerar la presencia de SM fue preciso presentar por lo menos tres de las condiciones citadas [8].

La investigación fue divulgada por teléfono a todas las unidades de salud vinculadas a la APS, informando las respectivas fechas de colecta de datos. Los cuestionarios fueron entregados a los profesionales, junto con el Término de Consentimiento Informado (TCI), por dos asistentes de investigación previamente preparados. Al final de cada colecta, los cuestionarios eran colocados en sobres individuales y codificados, para garantizar el sigilo y la confidencialidad.

Para la garantía de la homogeneidad en la aplicación de los cuestionarios, se realizó una calibración, en la cual uno de los asistentes aplicó los cuestionarios en 10 profesionales de la APS y 10 de otro sector y otro asistente reaplicó los cuestionarios a los mismos profesionales. Ese procedimiento fue realizado para calcular el índice de concordancia entre ellos, empleando el índice de Kappa, el cual tuvo el valor de 0,87, siendo considerado aceptable con clasificación de óptima [14].

Los datos fueron analizados con el uso del programa estadístico SPSS versión 20.0 de la Universidade do Estado da Bahia, Brasil (UNEB), y los resultados analizados por estadística descriptiva. Fueron estudiadas las asociaciones de cada variable, individualmente. La SB se presentó como la variable independiente y las condiciones metabólicas como la variable dependiente. La medida de asociación epidemiológica adoptada fue la Razón de Prevalencia (RP), siendo presentada por RP bruta.

Los preceptos éticos fueron seguidos integralmente. El presente estudio fue aprobado por el comité de Ética en Investigación involucrando seres humanos de la UNEB, bajo el parecer $n^{\circ}$ 872.365/2014.

Resultados

Se estudió la población de profesionales de enfermería de la APS, que correspondió al $95,2 \%$ de los individuos elegibles, siendo $23(38,3 \%)$ enfermeras y $37(61,7 \%)$ de auxiliares y técnicas de enfermería. Hubo $02(3,2 \%)$ rehúsas y $01(1,6 \%)$ de licencia médica. Entre los profesionales de enfermería estudiados $03(5 \%)$ eran del género masculino y 57 (95\%) femenino, con edad media de 39,5 años $( \pm 10,3)$. Sobre la situación marital, $34(56,7 \%)$ eran casadas y $26(43,3 \%)$ solteras, $38(63,3 \%)$ trabajaban hace 5 años o menos en la APS y 22 $(36,7 \%)$ hace más de 5 años, $14(23,3 \%)$ tenían otro vínculo de trabajo.

Las variables pertenecientes al estilo de vida presentan una frecuencia de $16(26,7 \%)$ para el consumo de bebidas alcohólicas, 40 (66,7\%) practicaban actividad física y $32(53,3 \%)$ se encontraban satisfechas con la forma física. En las variables biológicas, $49(84,5 \%)$ relataron problemas digestivos, $20(34,5 \%)$ mareos y $34(58,6 \%)$ dolores de cabeza.

Los datos de la Tabla II evidenciaron la relación entre las características socio demográficas, laborales, estilo de vida, biológicas y el SB. La prevalencia del SB fue 58,3\%, siendo que las mayores tendencias observadas fueron entre los hombres $(66,7 \%)$, jóvenes con hasta 40 años $(67,6 \%)$, los no negros $(60,9 \%)$, los sin hijos $(59,1 \%)$, con pareja $(64,7 \%)$ y con 1 a 2 sueldos mínimos de renta mensual $(65,3 \%)$. Entre estas características fueron encontradas asociaciones epidemiológicas positivas en la variable raza/color $(R P=1,22)$ e renta $(R P=1,20)$.

Sobre las características laborales, las mayores prevalencias del SB fueron entre los auxiliares y técnicos de enfermería $(62,2 \%)$, con más de 5 años de tiempo de trabajo $(68,2 \%)$ y con apenas un vínculo de trabajo $(65,2 \%)$. De estas, solo la variable categoría profesional presentó asociaciones epidemiológicas positivas $(\mathrm{RP}=1,19)$ (Tabla II). 
En la Tabla II también se observan las características del estilo de vida y las prevalencias de SB en cada nivel, con prevalencias mayores entre los profesionales de enfermería que no practicaban actividad física (70\%), que consumen bebida alcohólica (75\%) y que están satisfechos con la forma física (64,3\%). Se constataron asociaciones epidemiológicas positivas en todas ellas: práctica de actividad física, $(R P=1,33)$, consumo de bebida alcohólica $(R P=1,43)$ y satisfacción de la forma física $(R P=1,21)$.

Tabla II - Características socio demográficas, laborales, estilo de vida y biológicas de los profesionales de enfermería de la Atención Primaria de Salud de acuerdo con el diagnóstico de Síndrome de Burnout (N=60). Bahia, Brasil, 2015.

\begin{tabular}{|c|c|c|c|}
\hline \multirow{2}{*}{ Características } & \multicolumn{2}{|c|}{ Síndrome de Burnout } & \multirow[b]{2}{*}{$\mathbf{R P}$} \\
\hline & $\begin{array}{ll}\mathrm{Si} & (\mathrm{N}=35) \\
(58,3 \%) & \\
\mathrm{n}(\%) & \\
\end{array}$ & $\begin{array}{l}\text { No }(\mathrm{N}=25)(41,7 \%) \\
\text { n (\%) }\end{array}$ & \\
\hline \multicolumn{4}{|l|}{ Sociodemográficas } \\
\hline Femenino & $33(57,9)$ & $24(42,1)$ & 0,87 \\
\hline Masculino & $2(66,7)$ & $1(33,3)$ & 1,0 \\
\hline \multicolumn{4}{|l|}{ Edad } \\
\hline 40 años o más & $12(46,2)$ & $14(53,8)$ & 0,68 \\
\hline hasta 40 años & $23(67,6)$ & $11(32,4)$ & 1,0 \\
\hline \multicolumn{4}{|l|}{ Raza/color } \\
\hline Negros & $28(60,9)$ & $18(39,1)$ & 1,22 \\
\hline Blancos & $7(50)$ & $7(50)$ & 1,0 \\
\hline \multicolumn{4}{|l|}{ Hijos } \\
\hline $\mathrm{Si}$ & $22(57,9)$ & $16(42,1)$ & 0,98 \\
\hline No & $13(59,1)$ & $9(40,9)$ & 1,0 \\
\hline \multicolumn{4}{|l|}{ Estado civil } \\
\hline Sin pareja & $13(50)$ & $13(50)$ & 0,77 \\
\hline Con pareja & $22(64,7)$ & $12(35,3)$ & 1,0 \\
\hline \multicolumn{4}{|l|}{ Renta $^{*}$} \\
\hline De 1 a 2 sueldos mínimos & $18(64,3)$ & $10(35,7)$ & 1,20 \\
\hline Mayor o igual a 3 sueldos mínimos & $16(53,3)$ & $14(46,7)$ & 1,0 \\
\hline \multicolumn{4}{|l|}{ Laborales } \\
\hline \multicolumn{4}{|l|}{ Categoría profesional } \\
\hline Técnico (a) de enfermería & $23(62,2)$ & $14(37,8)$ & 1,19 \\
\hline Enfermero (a) & $12(52,2)$ & $11(47,8)$ & 1,0 \\
\hline \multicolumn{4}{|l|}{ Tiempo de trabajo } \\
\hline Menor o igual a 5 años & $20(52,6)$ & $18(47,4)$ & 0,77 \\
\hline Más que 5 años & $15(68,2)$ & $7(31,8)$ & 1,0 \\
\hline \multicolumn{4}{|l|}{ Otro vínculo de trabajo } \\
\hline $\mathrm{Si}$ & $5(35,7)$ & $9(64,3)$ & 0,56 \\
\hline No & $30(65,2)$ & $16(34,8)$ & 1,0 \\
\hline \multicolumn{4}{|l|}{ Estilo de vida } \\
\hline \multicolumn{4}{|l|}{ Práctica de actividad física } \\
\hline No & $14(70)$ & $6(30)$ & 1,33 \\
\hline \multirow{2}{*}{\multicolumn{4}{|c|}{ Consumo de bebida alcohólica }} \\
\hline & & & \\
\hline $\mathrm{Si}$ & $12(75)$ & $4(25)$ & 1,43 \\
\hline No & $23(52,3)$ & $21(47,7)$ & 1,0 \\
\hline \multicolumn{4}{|l|}{ Satisfecho con la forma física } \\
\hline No & $18(64,3)$ & $10(35,7)$ & 1,21 \\
\hline $\mathrm{Si}$ & $17(53,1)$ & $15(46,9)$ & 1,0 \\
\hline \multirow{2}{*}{\multicolumn{4}{|c|}{$\begin{array}{l}\text { Factores biológicos } \\
\text { Dolores de cabeza** }\end{array}$}} \\
\hline & & & \\
\hline $\mathrm{Si}$ & $22(64,7)$ & $12(35,3)$ & 1,41 \\
\hline No & $11(45,8)$ & $13(54,2)$ & 1,0 \\
\hline \multicolumn{4}{|l|}{ Mareos* } \\
\hline $\mathrm{Si}$ & $14(70)$ & $6(30)$ & 1,4 \\
\hline \multirow{2}{*}{\multicolumn{4}{|c|}{ Problemas digestivos* }} \\
\hline & & & \\
\hline $\mathrm{Si}$ & $4(44,4)$ & $5(55,6)$ & 0,75 \\
\hline No & $29(59,2)$ & $20(40,8)$ & 1,0 \\
\hline
\end{tabular}


Cuando se analizaron las características biológicas, las mayores prevalencias del SB fueron entre profesionales que relataron tener dolores de cabeza $(64,7 \%)$, mareos $(70 \%)$ y sin problemas digestivos $(59,2 \%)$, con asociaciones epidemiológicas positivas en las variables de dolores de cabeza $(\mathrm{RP}=1,41)$ y mareos $(\mathrm{RP}=1,4)$ (Tabla II).

En la Tabla III fueron presentadas las asociaciones del SB con las condiciones metabólicas. Dentro de los profesionales que fueron diagnosticados con SB 6,5\% tenían glicemia en ayuno alta, 32,3\% tenían triglicéridos altos, $14,3 \%$ con presión arterial sistólica alta y $20 \%$ con presión arterial diastólica alta, $54,3 \%$ con circunferencia abdominal aumentada y $28,6 \%$ con bajo HDL. Se observaron asociaciones epidemiológicas positivas con los triglicéridos $(R P=1,10)$ e HDL $(R P=1,02)$. La prevalencia del $S M$ fue de $30,9 \%$, se destacando más prevalencia en aquellos que no fue diagnosticado el SB (33,3\%), cuando se comparó a aquellos con el síndrome (29\%), con asociación epidemiológica negativa ( $R P=0,87)$ (Tabla III).

Tabla III - Asociaciones entre Síndrome de Burnout y Condiciones Metabólicas de los profesionales de enfermería de la Atención Primaria de Salud ( $N=60)$. Bahia, Brasil, 2015.

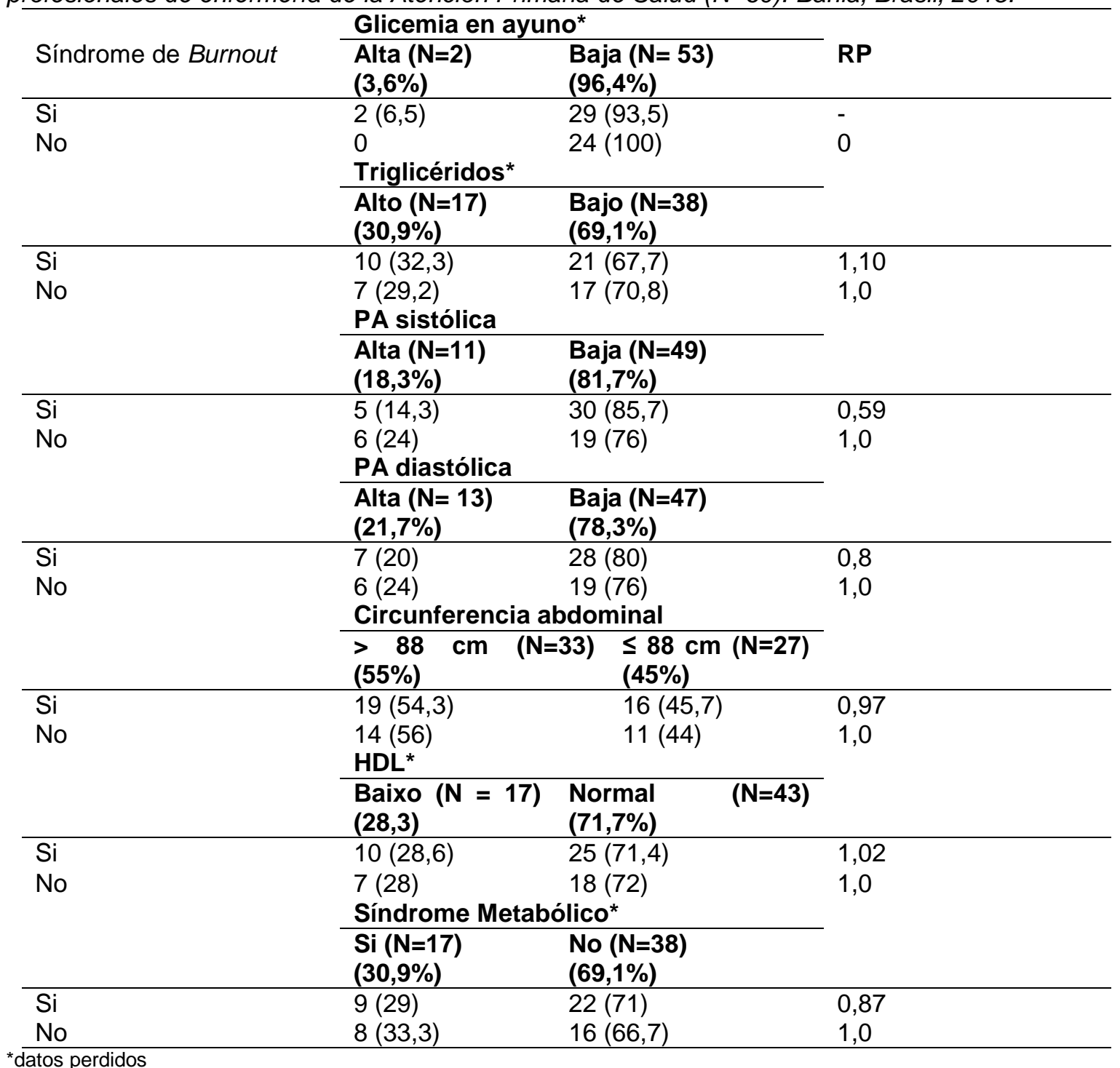

datos perdidos

Discusión

Este es el primer estudio piloto que investiga la relación entre Burnout y las condiciones metabólicas de los profesionales de enfermería de la APS. La muestra del estudio se constituyó por individuos jóvenes, predominantemente del género femenino, con pareja, cuya mayoría fue de técnicas de enfermería y con menos de cinco años de trabajo en la ABS. Se observó una alta prevalencia de acometimiento del SB entre los participantes de este estudio 
$(58,3 \%)$. Este resultado es correspondiente a lo que fue encontrado en investigaciones realizadas con profesionales de la APS, en las cuales las prevalencias encontradas se sitúan entre $10,6 \%$ y $39,3 \%[4,15]$. Debido a la utilización de los diversos instrumentos y escalas en los diferentes estudios, los valores pueden ser discrepantes, lo que provoca con que sea difícil la comparación entre ellos. A pesar de ello, en este estudio se consideran los índices de niveles significativos del SB.

Se notó que hubo más prevalencia de SB entre los hombres $(66,7 \%)$, conforme fue constatado en otras investigaciones [16-18]. Lo descubierto puede indicar que las mujeres buscan cuidar más de su salud mental de forma más frecuente e intentan combatir el estrés psicológico [15]. Hubo también una mayor prevalencia del Síndrome entre individuos con hasta 40 años de edad $(67,6 \%)$. Es interesante resaltar que las mayores prevalencias del SB encontradas en personas más jóvenes y con pocos años de experiencia profesional [19-21] pueden ser atribuidas al hecho de que la menor experiencia laboral puede generar una idealización y grandes expectativas de realización profesional, las cuales se pueden ver frustradas cuando no se concretan, desencadenando inseguridad, choque de realidad y decepciones. Estos factores vulnerabilizan la salud mental de los trabajadores, dejándolos susceptibles al SB [19,22].

Entre los participantes fueron evidenciadas asociaciones epidemiológicas positivas entre el SB y las variables raza/color de piel, categoría profesional y renta. No obstante, no fueron encontrados estudios en la literatura estudiada que las comparasen.

Con relación al estilo de vida, la mayoría de los participantes del estudio relató que no practica actividad física $(70,0 \%)$. Hace décadas que son creadas directrices nacionales e internacionales que recomiendan el mínimo de actividad física diaria para toda la población, considerando sus particularidades y los beneficios que estas pueden traer. Para la población adulta es recomendado realizar por lo menos 30 minutos de actividad física de intensidad moderada en la mayoría de los días de la semana [23,24]. En este sentido, se destacan los beneficios de los ejercicios físicos como eficaces para la reducción del estrés ocupacional [25]. La insatisfacción con la forma física declarada por $64,0 \%$ de los participantes puede estar relacionada con la falta de actividad física y consecuentemente la mayor probabilidad de sobrepeso y obesidad.

El consumo de bebidas alcohólicas fue mencionado por el $75 \%$ de los participantes. Estudios destacan que portadores de SB pueden hacer uso del consumo de bebidas alcohólicas como una estrategia de afrontamiento. Estos utilizan el alcohol como un medio regular y alternativo para lidiar con los sentimientos y pensamientos negativos, agotamiento psicológico y físico, los cuales son desencadenados por problemas en el ambiente de trabajo. Sin embargo, el consumo excesivo y frecuente de alcohol puede provocar dependencia química y otras anomalías sistémicas, lo que es altamente perjudicial al organismo [26-29].

En relación a los factores físicos, se encontraron asociaciones epidemiológicas positivas para dolores de cabeza $(R P=1,41)$ y mareos $(R P=1,40)$, los cuales son síntomas considerados como fuertes predictores del SB $[21,30]$.

Se consideró elevada la prevalencia de profesionales diagnosticados con el SM $(30,9 \%)$, aunque de estos, $33,3 \%$ no poseían el SB. Algunos estudios encontraron asociaciones positivas entre las condiciones cardiovasculares y el Burnout [31-36]. Otras investigaciones relatan asociación del SB con factores psicológicos, como depresión, sobrepeso y obesidad. En este sentido, la depresión es un índice de riesgo para el SM [37].

Frente a lo expuesto, se consideró elevada la prevalencia de profesionales diagnosticados con el SM (30,9\%), aunque de estos, 33,3\% no poseían el SB. Sin embargo, se observó una alta prevalencia del SB entre los participantes del estudio $(58,3 \%)$. Siendo así, es irrefutable la importancia que tienen las consecuencias fisiopatológicas del estrés agudo y crónico en la salud y calidad de vida de los profesionales de enfermería, sobre todo cuando se evalúa la influencia del mismo para el desarrollo del SB, lo que todavía puede resultar en alteraciones fisiológicas y neuroendocrinas, predisponiendo la ocurrencia de disturbios metabólicos, como es el caso del SM.

De esta manera, los desdoblamientos de este trabajo son notorios, visto la relevancia de los aspectos denotados. Con esto, todo el conocimiento que puede ser conjugado al que ya fue construido a la luz de la ciencia, podrá contribuir en nuevas estrategias de intervención, que 
deben ser implantadas con énfasis en los factores de riesgo emergentes, principalmente los psicosociales y del comportamiento.

1. Neves VF, Oliveira AF, Alves PC. Síndrome de Burnout: Impacto da satisfação no trabalho e da percepção de suporte organizacional. Psico 2014;45(1):45-54. http://dx.doi.org/10.15448/1980-8623.2014.1.12520.

2. Loures DP, Sant'Anna I, Baldotto CSR, Sousa ED, Nóbrega, ACL. Estresse mental e sistema cardiovascular. Arq Bras Cardiol 2002;78(5):525-30. http://dx.doi.org/10.1590/S0066-782X2002000500012

3. Ludwig MWB, Bortolon C, Bortolini M, Feoli AM, Macagnan FE, Oliveira MS. Ansiedade, depressão e estresse em pacientes com síndrome metabólica. Arq Bras Psico 2012;64 (1):31-46.

4. Merces MC, Silva DDE, Lua I, Oliveira DS, de Souza MC, D'Oliveira . Burnout syndrome and abdominal adiposity among Primary Health Care nursing professionals. Psicologia-Reflexo e Crítica 2016;29(44):2-8. http://dx.doi.org/10.1186/s41155-0160051-7

5. Sá MAS, Martins-Silva PO, Funchal B. Burnout: o impacto da satisfação no trabalho em profissionais de enfermagem. Psicol Soc 2014;26(3):664-74. http://dx.doi.org/10.1590/S0102-71822014000300015

6. Holmes ES, Santos SR, Farias JA, Costa MBS. Síndrome de burnout em enfermeiros na atenção básica: repercussão na qualidade de vida. Rev Pesqui Cuid Fundam 2014; 6(4):1384-1395. http://dx.doi.org/10.1590/S0102-71822014000300015

7. Melamed S, Shirom A, Toker S, Berliner S, Shapira I. Burnout and risk of cardiovascular disease: evidence, possible causal paths, and promising research directions. Psychol Bull 2006;132(3):327-53.

8. Sociedade Brasileira De Hipertensão. I Diretriz Brasileira de Diagnóstico e Tratamento da Síndrome Metabólica. Arq Bras Cardiol 2005;84(Supl1):3-28.

9. Oliveira C, Scarabelot VL, Cioato SG, Caumo W, Torres ILS. Inter-relação entre síndrome metabólica, estresse crônico e ritmos circadianos de marcadores adipogênicos: uma revisão. Rev HCPA 2013;33(3/4):257-68.

10. Costa MB, Guércio MNS, Costa HFC, Oliveira MME, Alves MJM. Possível relação entre estresse ocupacional e síndrome metabólica. HU Rev 2011;37(1):87-93.

11. Benevides-Pereira AMT. MBI - Maslach Burnout Inventory e suas adaptações para 0 Brasil. In: Anais da $32^{\underline{a}}{ }^{\mathrm{a}}$ Reunião Anual de Psicologia; 2001; Rio de Janeiro. p. 84-85.

12. Moreira DS, Magnago RF, Sakae TM, Magajewski FRL. Prevalência da síndrome de burnout em trabalhadores de enfermagem de um hospital de grande porte da Região Sul do Brasil. Cad Saúde Pública 2009;25(7):1559-68. http://dx.doi.org/10.1590/S0102311X2009000700014

13. Grunfeld E, Whelan TJ, Zitzelsberger L, Willan AR, Montesanto B, Evans WK. Cancer care workers in Ontario: prevalence of burnout, job stress and job satisfaction. CMAJ 2000;163(2):166-9.

14. Seigel DG, Podgor MJ, Remaley NA. Acceptable values of kappa for comparison of two groups. Am J Epidemiol 1992;135(5):571-8.

15. Navarro-González D, Ayechu-Díaz A, Huarte-Labiano I. Prevalencia del síndrome del burnout y factores asociados a dicho síndrome en los profesionales sanitarios de Atención Primaria. Semergen 2015;41(4):191-8. https://doi.org/10.1016/j.semerg.2014.03.008

16. Prieto-Albino L. Burnout among primary care physicians. Aten Primaria 2001;28:444-5.

17. Sibbald B, Enzer I, Cooper C, Rout U, Sutherland V. GP job satisfaction in 1987, 1990 and 1998: Lessons for the future? Fam Pract 2000;17(5):364-71.

18. Goehring C, Bouvier Gallacchi M, Künzi B, Bovier P. Psychosocial and professional characteristics of burnout in Swiss primary care practitioners: a cross-sectional survey. Swiss Med Wkly 2005;135(7-8):101-8.

19. Benevides-Pereira AMT. Burnout: quando o trabalho ameaça o bem-estar do trabalhador. São Paulo: Casa do Psicólogo; 2002.

20. Aranda-Beltrán C, Pando-Moreno M, Torres-López T, Estrada-Salazar J, FrancoChávez S. Factores psicosociales y síndrome de burnout en médicos de familia. México. An Fac Med Lima 2005;66(3):225-31. 
21. Maslach C, Schaufeli WB, Leiter MP. Job Burnout. Annu Rev Psycol 2001;52:397-422.

22. Carlotto MS. Síndrome de Burnout: um tipo de estresse ocupacional. Canoas: ULBRA; 2001.

23. Prentice AM. The emerging epidemic of obesity in developing countries. Int $\mathrm{J}$ Epidemiology 2006;35(1):93-9.

24. Yach D, Stuckler D, Brownell KD. Epidemiologic and economic consequences of the global epidemics of obesity and diabetes. Nat Med 2006;12:62-6.

25. Conn VS, Hafdahl AR, Cooper PS, Brown LM, Lusk SL. Meta-analysis of workplace physical activity interventions. Am J Prev Med 2009;37(4):330-9.

26. Frone MR. Work stress and alcohol use. Alcohol Res Health, 1999;23(4):284-91.

27. Rhem J, Gmel G, Sempos CT, Trevisan M. Alcohol-related morbidity and mortality. Alcohol Research \& Health 2002;27(1):39-51.

28. Carlini EA. Epidemiologia do uso do álcool no Brasil. Arq Méd ABC 2006(Supl2):4-7.

29. Maslach C. Burnout: The Cost of Caring. Cambridge: Malor Books; 2003.

30. Maslach C, Jackson SE. The measurement of experienced burnout. J Occup Behaviour 1981;2:99-113.

31. Appels A, Falger PRJ, Schouten EGW. Vital exhaustion as risk indicator for myocardial infarction in women. J Psychosom Res 1993;37(8):881-90.

32. Appels $A$, Mulder $P$. Excess fatigue as a precursor of myocardial infarction. Eur Heart $J$ 1998;9:758-64.

33. Schuitemaker GE, Dinant GJ, Van Der Pol GA, Appels A. Assessment of vital exhaustion and identification of subjects at increased risk of myocardial infarction in general practice. Psychosomatics 2004;45(5):414-8.

34. Schuitemaker GE, Dinant GJ, Van Der Pol GA, Verhelst AFM, Appels A. Vital exhaustion as a risk indicator for first stroke. Psychosomatics 2004;45(2):114-8.

35. Prescott E, Holst C, Gronbaek M, Schnohr P, Jensen G, Barefoot J. Vital exhaustion as a risk factor for ischaemic heart disease and all-cause mortality in a community sample. A prospective study of 4084 men and 5479 women in the Copenhagen City Heart Study. Int J Epidemiol 2003;32(6):990-7.

36. Cole SR, Kawachi I, Sesso HD, Paffenbarger RS, Lee IM. Sense of exhaustion and coronary heart disease among college alumni. Am J Cardiol 1999;84(2):1401-5.

37. Goldbacher EM, Bromberger J, Matthews KA. Lifetime history of major depression predicts the development of the metabolic syndrome in middle-aged women. Psychosoma Med 2009;71(3):266-72. http// doi: 10.1097/PSY.0b013e318197a4d5 . 